\title{
Clinical Characteristics of Metastatic Gastric Tumors: A Report of 8 Cases in a Single Hospital
}

\author{
Jin Bo ${ }^{*}$, Liu Yan-ping ${ }^{2}$ and Wang Hai-jiang ${ }^{1}$ \\ ${ }^{1}$ Department of Gastroenteropathy Surgery, The Third Affiliated Hospital (Xinjiang Tumor Hospital), Xinjiang \\ Mediacl Universituy, Ulumuqi, Xinjiang, China \\ ${ }^{2}$ Dpartment of Anesthesiology, The Third Affiliated Hospital (Xinjiang Tumor Hospital), Xinjiang Mediacl \\ Universituy, Ulumuqi, Xinjiang, China
}

${ }^{*}$ Corresponding author: Jin Bo, Department of Gastroenteropathy Surgery, The Third Affiliated Hospital (Xinjiang Tumor Hospital), Xinjiang Mediacl Universituy, Ulumuqi 830011, Xinjiang, China, Tel: +86-9917819101, E-mail: aupt1980@sina.com

Citation: Jin Bo, Liu Yan-ping, Wang Hai-jiang (2014) Clinical Characteristics of Metastatic Gastric Tumors: A Report of 8 Cases in a Single Hospital. J Case Rep Stud 2(2): 203. doi: 10.15744/2348-9820.1.503

Received Date: April 16, 2014 Accepted Date: April 28, 2014 Published Date: April 30, 2014

\begin{abstract}
Metastatic gastric tumors (MGTs) mean the tumor cells that attack the stomach and grow there through blood vessel, lymph vessel, and other pathway, consistent with the primary tumor in phenotype, which are clinically uncommon, and information on MGTs is generally limited to single case reports. Here we present a clinical series of 8 cases with MGTs, in attention to discuss the clinical characteristics, diagnosis and treatment, and prognosis of MGTs. Our data showed that MGTs are rare, with a male predominance, and the cause of death was multiple organ metastases in most cases. Heterochromous MGTs showed a significantly better prognosis than simultaneous MGTs, and a long interval between initial radical excision of the primary tumor and appearance of gastric metastasis was found to be associated with good prognosis.
\end{abstract}

\section{Introduction}

Metastatic gastric tumor (MGT), namely secondary gastric tumor, means the tumor cells that attack the stomach and grow there through blood vessel, lymph vessel, and other pathway, consistent with the primary tumor in phenotype, whereas the canceration of ectopic tissue such as ectopic pancreas, however, belongs not to MGT, because of its primary characteristic [1]. As gastric metastases are clinically uncommon, information on MGTs is generally limited to single case reports. Here we present a clinical series of 8 cases with MGTs, in attention to discuss the clinical characteristics, diagnosis and treatment, and prognosis of MGTs.

\section{Clinical data}

Between January 1990 and December 2012, a total of 6940 patients with gastric tumors in our hospital disclosed 8 cases of MGTs. The primary tumors were adenocarcinoma of distal esophagus (2 cases), melanoma in foot ( 2 cases), hepatocellular carcinoma ( 1 case), small-cell undifferentiated carcinoma of lung (1 case), clear-cell carcinoma of kidney ( 1 case), and atypical medullary carcinoma of breast ( 1 case). The diagnosis was made based on findings in the endoscopic or surgical specimen, and patients with malignant lymphoma or direct invasion from adjacent organs were excluded. Of these, 3 were discovered in surgical specimens and 5 at autopsy. MGTs were detected simultaneously with the primary tumors in 6 and afterward in 2 patients at 24 and 72 months, respectively. Multiple organ metastases were present simultaneously in 2 patients. Although 6 patients underwent gastrectomy, macroscopic eradication of MGT was accomplished in only 4, of whom a UICC R0 resection was possible in only 3. Clinical data of each case was listed in Table 1-3.

\section{Results from the cases}

According to the data in our hospital, MGTs had a highly low incidence, accounting for only $0.12 \%$ (8/6490) of gastric tumors. The gender ratio (male/female) was 3:1 (6:2), suggesting a male predominance, while the median age of the patients was 58.5 years (range, 38-74). The data showed a life span from 2 months to over 10 years, with a median life span of 8.5 months.

The clinical characteristic was major in nonspecific digestive symptoms such as epigastralgia, melena, hematemesis, anemia, dysphagia, etc, usually difficult to discriminate from primary gastric carcinoma. The final diagnosis of gastric tumor was based on the pathology of the gastrectomy specimen in 4 patients and by endoscopic biopsy in all 8 cases. The tumor size ranged from 10 to $72 \mathrm{~mm}$ (median $19.5 \mathrm{~mm}$ ). The metastatic site tend to be the upper stomach, when the primary site was esophagus, while other primary tumors resulted in the middle or lower stomach as the metastatic site. 


\begin{tabular}{|c|c|c|c|c|c|c|}
\hline Patient No. & Sex & Age & Nationality & Primary organ & Histologic type & Treatment for primaries \\
\hline 1 & M & 70 & Han & Esophagus & Moderately differntiated adenocarcinoma & neoadjuvant chemotherapy + Resection \\
\hline 2 & M & 38 & Kazak & Esophagus & Signet ring cell carcinoma & Resection \\
\hline 3 & M & 47 & Han & Foot skin & Melanoma & No \\
\hline 4 & $\mathrm{~F}$ & 58 & Han & Foot skin & Melanoma & No \\
\hline 5 & M & 43 & Han & Liver & Hepatocellular carcinoma & Chemotherapy \\
\hline 6 & M & 74 & Han & Lung & Small-cell undifferentiated carcinoma & Chemotherapy \\
\hline 7 & M & 66 & Uygur & Kidney & Clear-cell carcinoma & Resection \\
\hline 8 & $\mathrm{~F}$ & 59 & Han & Breast & Atypical medullary carcinoma & Resection + Chemotherapy \\
\hline
\end{tabular}

Table 1: Cliniopathological findings of primary tumors

\begin{tabular}{|c|c|c|c|c|c|c|c|}
\hline Patient No. & Symptom & MGT-free interval(Months) & Location (third) & Size(mm) & T & N & Serum CEA level (ng/ml) \\
\hline 1 & Dysphagia & 0 & upper & 15 & 3 & 3 \\
\hline 2 & Hematemesis & 0 & upper & 21 & 4 & 3 & 7.416 \\
\hline 3 & Anemia & 0 & middle + lower & 72 & 4 & 2 & 130.579 \\
\hline 4 & Epigastralgia & 0 & lower & 18 & 3 & 2 \\
\hline 5 & Epigastralgia & 0 & middle & 40 & 4 & 1 & 3.974 \\
\hline 6 & Melena & 0 & middle & 10 & 3 & 0 \\
\hline 7 & Melena & 72 & lower & 13 & 2 & 0 & 3.871 \\
\hline 8 & Epigastralgia & 24 & lower & 24 & 4 & 1 \\
\hline
\end{tabular}

Table 2: Cliniopathological findings of metastatic gastric tumors (MGTs)

\begin{tabular}{|c|c|c|c|c|c|}
\hline Patient No. & $\begin{array}{c}\text { Gastrectomy } \\
\text { (Curability) }\end{array}$ & Chemotherapy & Simultaneous metastatic site & $\begin{array}{c}\text { Survival after MGT } \\
\text { (days) }\end{array}$ & Cause of death \\
\hline 1 & Cure (B) & Yes & No & 1096 & Petitoneal metastases \\
\hline 2 & Cure (C) & No & No & 98 & Multiple organ metastases \\
\hline 3 & No & No & Liver, bone lung & 95 & Multiple organ metastases \\
\hline 4 & No & No & No & 158 & Multiple organ metastases \\
\hline 5 & No & No & No & 365 & Lung cancer \\
\hline 6 & No & No (Interferon) & No organ metastases \\
\hline 7 & Cure (B) & Yes & No & Over 3 years & No \\
\hline 8 & Cure (B) & & & No \\
\hline
\end{tabular}

B: No residual disease

C: Definite residual disease

Table 3: Clinical course after detection of MGT

CT scan were useful for noninvasive assessment for estimation of the degree of tumor penetration through the gastrointestinal wall. The serum carcino-embryonic antigen (CEA) level was usually higher with a primary adenocarcinoma of esophagus, whereas the contrary condition was shown with other primary tumors.

Simultaneous MGT was diagnosed when the primary tumor was adenocarcinoma of distal esophagus, melanoma, hepatocellular carcinoma, or small-cell undifferentiated carcinoma of lung, while heterochromous MGT was diagnosed with the primary tumor of clear-cell carcinoma of kidney or atypical medullary carcinoma of breast, respectively in 6 years and 2 years after surgery, both cases as old patients, who received adjuvant treatment after surgery, and the primary tumor didn't recur at the same site but resulting in distant metastasis. 6 cases had solitary gastric metastasis and 2 cases had metastases in other locations including liver, bone, lung, etc. There were no significant differences in age, gender, nationality, or tumor location between solitary metastasis and multiple metastases. Heterochromous MGTs showed a significant better prognosis than simultaneous MGTs, and a long interval between initial radical excision of the primary tumor and appearance of gastric metastasis was found to be associated with better outcome. The cause of death was multiple organ metastases in most cases. 
4 patients underwent gastrectomy with intent to cure, but R0 resection was possible in 3 patients and their survival was more than 3 years (case 1, 7 and 8). In contrast, 1 patient (case 2) who underwent palliative gastrectomy survived for only 98 days. Although MGTs suggested a terminal stage of tumor, a radical gastrectomy resulted in a better prognosis for resectable cases, especially for heterochromous MGTs, with a life span from 3 years to over 10 years. 4 patients were treated by chemotherapy (case 1, 5, 6, 8), with survival time from 158 days to more than 3 years. The longest survival in our series was over 10 years after gastrectomy with adjunctive therapy of interferon in case 7.

\section{Disscusson}

It's not uncommon for the stomach to be directly attacked by the carcinoma of organ close to the stomach such as hepatic cancer, cholangiocarcinoma, panreatic cancer, colon cancer, etc. But based on clinical and autopsy findings, the stomach was an unusual site for distant metastasis, and the reported incidence of MGTs was $0.2-1.7 \%$ [2-4], higher than our results (0.12\%). Reports on this subject were poor and often limited to single case reports, so metastatic involvement of the stomach was not well known. The incidence of MGTs in autopsy studies had been reported to be as high as 15\% from breast cancer [5], 9\% from lung cancer [6], 9\% from esophageal cancer [7], and 23\% from melanoma [8]. Our results suggested that MGTs originated from esophageal carcinoma and melanoma more commonly. The stomach receives a rich blood supply, so hematogenous metastasis sounds more possible [4]. Tumor cells in the blood could be trapped in the submucosal layer of the stomach and develop as submucosal tumors [2], and the localization could also simulate a primary gastric cancer, and any region of gastrointestinal tract, from the tongue [5] to the anum [9], could be involved.

Absent of special clinical characteristic, it's difficult to discriminate between MGT and primary gastric carcinoma. Thus, the diagnosis of MGTs is difficult because of its asymptomatic and nonspecific symptoms, which could be modified by the primary disease and the effects of treatment such as chemotherapy. Clinically, the diagnosis of MGTs depends on the primary tumor history and histopathological examination. It was reported that MGTs were often detected at an advanced stage of the disease [4]. Gastroendoscopy usually disclosed a firm elevated lesion with central irregular erosion [10]. Although histologic confirmation of the endoscopic specimen is difficult [11-15], endoscopy with biopsy is mandatory to perform a definite diagnosis, and it was emphasised by Ambroggi [16] that endoscopy remains today the best diagnostic method to evaluate pathology of the upper gastrointestinal tract, in spite of that in some conditions endoscopy can allow false negative results, especially when the tumor is submucosal. Endoscopy can be repeated or combined with endoscopic ultrasound (EUS). Frequent endoscopic patterns include volcano-like ulcers, multiple nodules bull's eye appearance, extrinsic mass lesions, ulceration and polypoid tumor masses [14,17].

To choose a effective scheme is difficult, especially for neoadjuvant chemotherapy, so surgery should be made a priority for consideration, although long-term survival is rare [18]. And surgery is often needed for diagnosis and treatment of complications, like intestinal obstruction or bleeding [16]. Because multiple metastases are common, the prognosis of MGT is poor even after curative resection, with a median survival of less than 12 months $[7,14]$.

In our data, 4 patients underwent gastrectomy, of whom R0 resection was achieved in 3 patients with survival of more than 3 years (case 1, 7 and 8), suggesting a survival benefit. In contrast, 1 patient (case 2) who underwent palliative gastrectomy survived with tumor for only 98 days. So our results indicated that R0 resection was associated with better prognosis, especially to heterochromous MGTs (3-10 years). Basu et al. [19] advocated systemic therapy for gastric metastases from breast cancer, and Ihde and Coit. [20] recommended that palliative surgery be considered for MGTs from melanoma because of the low operative mortality and morbidity associated with it. Chemotherapy or hormonal treatment may result in fair palliation in selected patients, but it is important to be aware of bleeding and perforation during systemic chemotherapy [21]. Moreover, the evaluation of these few clinical cases showed that chemotherapeutic treatment of MGTs does not differ from that of metastases in other sites [22]. 4 patients with MGTs in our hospital received chemotherapy (case 1, 5, 6,8), with survival time from 158 days to more than 3 years, of whom 2 patients who underwent R0 resection resulted in better prognosis, so we thought that if chemotherapy contributed to $\mathrm{R} 0$ resection, it might contribute to better prognosis.

Of MGTs from esophageal carcinoma, primary lesions were most frequently located in the middle of thoracic esophagus, and lymphatic invasion, intramural metastases within the esophagus, as well as lymph node metastases, were predominant, and Saito et al. [7] suggested that surgical resection be performed when MGT from esophageal cancer was limited to the stomach and $<2$ $\mathrm{cm}$. MGTs are mainly hematogenous [23], whereas a gastric metastasis from esophageal cancer may occur through the submucosal longitudinal lymphatic network [7]. 2 patients (case 1 and 2) with MGTs from esophageal carcinoma in our data were male, with location of MGTs in upper stomach and higher serum CEA level. As simultaneous MGT, because of close distance between MGT and primary tumor, surgery was performed, but both patients died of distant metastasis to other organs.

Breast cancer is the most common tumor in women [24], and common sites of breast cancer metastasis include lungs, liver, bones, soft tissue, brain, and adrenal glands [16]. Reviewing the literature, gastrointestinal metastases from breast cancer are rare, and among the fragmentary reports, the most frequent site of metastasis was the stomach $(60 \%)$, and the recurrence could be very late [16]. Taal et al. [14] reported that 10 of 51 patients with gastric metastases from breast carcinoma had negative endoscopic biopsies, and Oda et al. [25] found that endoscopic findings of MGTs from breast cancer resembled type 4 advanced gastric cancer. 
Most series reported a greater propensity for lobular carcinoma rather than ductal breast carcinoma to metastasize to the gastrointestinal tract $[14,26]$. The reason is not clear, and some authors think that it might be related to a particular tropism of lobular cells $[27,28]$. It was advocated that MGTs from breast cancer is evidence of a systemic disease and therefore systemic therapy, such as chemotherapy and/or hormonal therapy, was advised [14]. Zelek et al. [29] reported a median survival of 21 months for patients who received chemotherapy, whereas it was less than 12 months for those who underwent gastrectomy for MGT from breast cancer. Here we presented a case (case 8) of heterochromous MGT from atypical medullary carcinoma of breast, which had never been reported, to our knowledge. The patient received neoadjuvant chemotherapy plus R0 recection, and lived for over 3 years till today.

Gastrointestinal tract is rare site of metastasis of renal cell carcinoma, besides thyroid, pancreas, skeletal muscles and skin [30, 31]. A report of 22 patients showed that the median time interval from radical excision of the primary tumor to the detection of gastric metastasis (IGM) was 6.3 years, and patients with an IGM $<6.3$ years had a significantly poorer prognosis than those with an IGM $\geq 6.3$ years, while patients with solitary gastric metastasis arising from renal cell carcinoma had better outcomes following treatment compared with those with multiple metastases [18]. These results suggested that since MGTs arising from renal cell carcinoma and breast cancer were slow growing, there might be similarities in the clinical characteristics associated with these cancers. Molecular agents targeting metastatic renal cell carcinoma, including sorafenib and sunitinib, were reported to possess the potential to improve progression-free survival, objective response rate and quality of life [32]. There was one case of heterochromous MGT from clear-cell carcinoma of kidney in our series (case 7), who received R0 recection plus adjunctive therapy of interferon, resulting in survival time of more than 10 years as a cure.

About MGT from lung cancer, it was reported that the most common histologic type of lung tumor was squamous cell (33\%), followed by large cell (29\%), and oat cell (19\%), and the esophagus was a more common site of involvement than stomach [6]. Dysphagia was the most common symptom when the tumor involved stomach, and it was usually radiographically demonstrated as "bulls eye" or "target" lesions of the stomach [33]. Besides hemotogenous metastasis, the possibility that sputum with cancer cells implanted into the stomach should not be excluded. In our series, a patient (case 6) with simultaneous MGT from small-cell undifferentiated carcinoma of lung received chemotherapy for 1 year but finally died of lung cancer, resulting in a bad prognosis.

Oda et al. [25] reported that malignant melanoma was the most common tumor to metastasize to the stomach. The incidence of clinical MGTs from cutaneous melanoma was reported to be $2.1 \%$ [4]. The majority of gastric metastases arising from malignant melanoma were detected within 2 years, most likely due to the rapid progression of the tumor [3,17]. But Pector et al. [34] reported one case of a patient who had been alive more than 6 years after subtotal gastrectomy for MGT from malignant melanoma. Our data included 2 cases (case 3 and 4) of simultaneous MGT from melanoma in foot, because of simultaneous multiple organ metastases and absent of effective treatment, both patients died in 3 months.

MGT from hepatic carcinoma was clinically uncommon, and it should be imageologically discriminated among MGT from he -patic carcinoma, hepatic metastasis from gastric carcinoma, and double primary carcinoma of both stomach and liver. History of hepatitis, higher serum a-fetoprotein (AFP) level, and multiple lesion within liver, might be helpful to discrimination. Pathologically, MGT from hepatic carcinoma has to be discriminated from gastric hepatoid adenocarcinoma. Quinquagenarian, common location in gastric antrum, higher serum AFP level, significant hepatoid differentiation of carcinoma tissue, early happenning of hepatic metastasis, and bad prognosis, were reported to be characteristic of gastric hepatoid adenocarcinoma [35,36]. We reported here a case of MGT from hepatocellular carcinoma, who lived for only 158 days after chemotherapy.

In conclusion, MGTs are rare, with a male predominance, and the common cause of death was multiple organ metastases. Heterochromous MGTs showed a significantly better prognosis than simultaneous MGTs, and a long interval between initial radical excision of the primary tumor and appearance of gastric metastasis was found to be associated with good outcome. Thus, redical treatment such as surgery should be considered for heterochromous MGTs.

\section{References}

1. Jin B, Liu YP, Wang HJ, Wang QS (2012) Gastric metastasis of atypical medullary carcinoma from breast: a case report. Chin Med Sci J 27: 121-4.

2. Menuck LS, Amberg JR (1975) Metastatic disease involving the stomach. Am J Dig Dis 20: 903-13.

3. Green LK (1990) Hematogenous metastases to the stomach. A review of 67 cases. Cancer 65: 1596-600.

4. Kobayashi O, Murakami H, Yoshida T, Cho H, Yoshikawa T, et al. (2004) Clinical diagnosis of metastatic gastric tumors: clinicopathologic findings and prognosis of nine patients in a single cancer center. World J Surg 28: 548-51.

5. Cormier WJ, Gaffey TA, Welch JM, Welch JS, Edmonson JH (1980) Linitis plastica caused by metastatic lobular carcinoma of the breast. Mayo Clin Proc 55: 747-53.

6. Antler AS, Ough Y, Pitchumoni CS, Davidian M, Thelmo W (1982) Gastrointestinal metastases from malignant tumors of the lung. Cancer 49: 170-2.

7. Saito T, Iizuka T, Kato H, Watanabe H (1985) Esophageal carcinoma metastatic to the stomach. A clinicopathologic study of 35 cases. Cancer 56 : $2235-41$.

8. Patel JK, Didolkar MS, Pickren JW, Moore RH (1978) Metastatic pattern of malignant melanoma. A study of 216 autopsy cases. Am J Surg 135: 807-10.

9. Puglisi M, Varaldo E, Assalino M, Ansaldo G, Torre G, et al. (2009) Anal metastasis from recurrent breast lobular carcinoma: a case report. World J Gastroenterol 15: $1388-90$. 
10. Konishi H, Isetani K, Satoh T, Fukuda S, Kodama T, et al. (1994) A case of metastatic chondrosarcoma of the stomach. J Gastroenterol 29: 495-500.

11. Taylor RR, Phillips WS, O’Connor DM, Harrison CR (1994) Unusual intramural gastric metastasis of recurrent epithelial ovarian carcinoma. Gynecol Oncol 55: $152-5$.

12. Lorimier G, Binelli C, Burtin P, Maillart P, Bertrand G, et al. (1998) Metastatic gastric cancer arising from breast carcinoma: endoscopic ultrasonographic aspects. Endoscopy 30: 800-4.

13. Odori T, Tsuboi Y, Katoh K, Yamada K, Morita K, et al. (1998) A solitary hematogenous metastasis to the gastric wall from renal cell carcinoma four years after radical nephrectomy. J Clin Gastroenterol 26: 153-4.

14. Taal BG, Peterse H, Boot H (2000) Clinical presentation, endoscopic features, and treatment of gastric metastases from breast carcinoma. Cancer 89: $2214-21$.

15. Wang MH, Wu MS, Wang HP, Change YL, Lin JT (2000) Endoscopic manifestations of metastatic hepatocellular carcinoma to the stomach: report of two cases. Gastrointest Endosc 52: 273-5.

16. Ambroggi M, Stroppa EM, Mordenti P, Biasini C, Zangrandi A, et al. (2012) Metastatic breast cancer to the gastrointestinal tract: report of five cases and review of the literature. Int J Breast Cancer 2012:439023.

17. De Palma GD, Masone S, Rega M, Simeoli I, Donisi M, et al. (2006) Metastatic tumors to the stomach: clinical and endoscopic features. World J Gastroenterol 12: $7326-8$.

18. Namikawa T, Munekage M, Kitagawa H, Okabayashi T, Kobayashi M, et al. (2012) Metastatic gastric tumors arising from renal cell carcinoma: Clinical characteristics and outcomes of this uncommon disease. Oncol Lett 4: 631-6.

19. Basu S, Last A, Shinkfield M (2002) Invasive lobular carcinoma of the breast presenting successively with colonic and gastric metastases. Int J Clin Pract 56: $623-5$.

20. Ihde JK, Coit DG (1991) Melanoma metastatic to stomach, small bowel, or colon. Am J Surg 162: 208-11.

21. Suzaki N, Hiraki A, Ueoka H, Aoe M, Takigawa N, et al. (2002) Gastric perforation due to metastasis from adenocarcinoma of the lung. Anticancer Res 22: $1209-12$.

22. Catino A, Lorusso V, Gargano G, Pellecchia A, Marzullo F, et al. (1992) Metastatic involvement of the stomach secondary to breast carcinoma. A case report. Eur J Gynaecol Oncol 13: 85-8.

23. Meyers MA, McSweeney J (1972) Secondary neoplasms of the bowel. Radiology 105: 1-11.

24. Schwarz RE, Klimstra DS, Turnbull AD (1998) Metastatic breast cancer masquerading as gastrointestinal primary. Am J Gastroenterol 93: 111-4.

25. Oda, Kondo H, Yamao T, Saito D, Ono H, et al. (2001) Metastatic tumors to the stomach: analysis of 54 patients diagnosed at endoscopy and 347 autopsy cases. Endoscopy 33: 507-10.

26. Borst MJ, Ingold JA (1993) Metastatic patterns of invasive lobular versus invasive ductal carcinoma of the breast. Surgery 114: 637-41.

27. Nazareno J, Taves D, Preiksaitis HG (2006) Metastatic breast cancer to the gastrointestinal tract: a case series and review of the literature. World J Gastroenterol 12: $6219-24$

28. McLemore EC, Pockaj BA, Reynolds C, Gray RJ, Hernandez JL, et al. (2005) Breast cancer: presentation and intervention in women with gastrointestinal metastasis and carcinomatosis. Ann Surg Oncol 12: 886-94.

29. Zelek L, Cottu PH, Mignot L, de Roquancourt A, Fizazi K, et al. (2001) Gastric metastases from breast cancer: a retrospective series of 12 patients. Am J Clin Oncol 24: 363-5.

30. Pollheimer MJ, Hinterleitner TA, Pollheimer VS, Schlemmer A, Langner C (2008) Renal cell carcinoma metastatic to the stomach: single-centre experience and literature review. BJU Int 102: 315-9.

31. Sadler GJ, Anderson MR, Moss MS, Wilson PG (2007) Metastases from renal cell carcinoma presenting as gastrointestinal bleeding: two case reports and a review of the literature. BMC Gastroentero7: 4.

32. Motzer RJ, Michaelson MD, Rosenberg J, Bukowski RM, Curti BD, et al. (2007) Sunitinib efficacy against advanced renal cell carcinoma. J Urol 178: 1883-7.

33. Rubin SA, Davis M (1985) "Bull's eye" or "target” lesions of the stomach secondary to carcinoma of the lung. Am J Gastroenterol 80: 67-9.

34. Pector JC, Crokaert F, Lejeune F, Gerard A (1988) Prolonged survival after resection of a malignant melanoma metastatic to the stomach. Cancer 61: 2134-5.

35. Roberts CC, Colby TV, Batts KP (1997) Carcinoma of the stomach with hepatocyte differentiation (hepatoid adenocarcinoma). Mayo Clin Proc 72: 1154-60.

36. Chang YC, Nagasue N, Kohno H, Taniura H, Uchida M (1990) Clinicopathologic features and long-term results of alpha-fetoprotein-producing gastric cancer. Am J Gastroenterol 85: 1480-5.

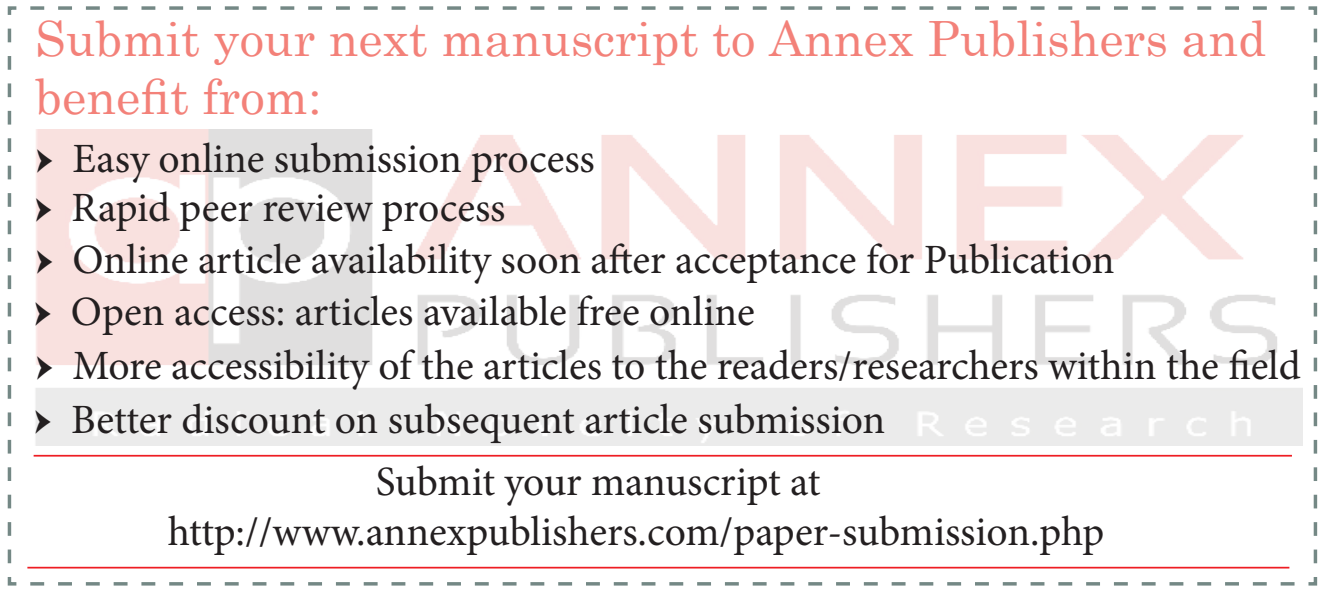

